\title{
LONG-RANGE MONITORING SYSTEM OF IRRIGATED AREA BASED ON MULTI-AGENT AND GSM
}

\author{
Tinghong Zhao ${ }^{1}$, Zibin Man $^{2}$, Xueyi Qi ${ }^{1, *}$ \\ ${ }^{I}$ College of Fluid Power and Control, Lanzhou University of Technology, Gansu, Chinan, \\ 730050 \\ ${ }^{2}$ Laboratory, Lanzhou University of Technology, Gansu, China, 730050 \\ * Corresponding author, Address: P.O. Box 53, College of Fluid Power and Control \\ Engineering, Lanzhou University of Technology, Gansu, 730050, P. R. China, Tel: +86- \\ 931-2976770, Fax:+86-931-2976750, Email: zhaoth2626@163.com
}

Abstract: In order to improve irrigated automatic degree of irrigated area, and make the control center and telemeter station to intelligent complete the task, structures the control center and telemeter station Agent union by instruct Multi-Agent theory, and make use of GSM network to finish the communication between the control center and telemeter station, at last make up the long-range monitoring system of irrigated area based on Multi-Agent and GSM. In this system, telemeter station finishes its functions, such as collection, memory, dealing with of the data, and while to carry control out according to the treated data, send the data that the control center needed to the control center Agent union through GSM network; control center Agent union store and deal with the accepted data information from telemeter station at first, then send the treated data to telemeter station Agent union to carry out new regulation and running. The setting-up of this system, not only make the work of the control center and telemeter station have higher intelligent, but also make the communication amount reduce greatly, communication is swifter and highefficient, which make the real-time character of long-range controls improve greatly.

Keywords: Multi-Agent; GSM network; Long-range monitoring system of irrigated area; Agent union; 


\section{INTRODUCTION}

With the constant development of the technology of computer and network, it is easier and easier to realize irrigated area long-range monitoring. The wired network is fast and swift in communication, used widely in a lot of fields, but for the scattering of distribution of irrigated area telemeter station, very difficult to wiring, so is less applied in the long-range monitoring system of irrigated area. Modern wireless communication network GSM is relatively suitable in the long-range monitoring system of irrigated area, and it has got certain application in long-range monitoring of irrigated area and has got better effect. (Zhang, 2005)

However, as to long-range monitoring system of irrigated area, GSM network is only a communication media, only play a role in transmitting information of monitoring and controlling, so in the monitoring system, most work need staff member to finish. To reduce the staff member participated and easily control, these works only can finish in monitor center. Thus, telemeter station has accurately and swiftly telemeter, but the course that telemeter station send telemeter message to monitor center through GSM network, which is analyzed by monitor and sent back telemeter by through GSM network, need longer time, make the real-time character of the longrange monitoring system of irrigated area relatively bad.

In order to change this kind of phenomenon, this paper combines MultiAgent theory and GSM network together, set up a kind of new-type longrange monitoring system of irrigated area. In this system, structure a Agent union respectively in monitor center and telemeter station, through defining structure, function etc. of each one Agent inside the union, to make them have certain intelligence, they can finish one's own task through one's own intellectual behavior. The monitor center Agent union finish such functions as the dispatching calculate of irrigation water of the whole irrigated area, storing dispatching information and sending control information etc. through the negotiation among inside Agent. Telemeter station Agent union is used to finish such functions of monitoring in real time, dealing with and calculation of monitoring information, analysis and storing of calculation result etc. confirm one's own control task in time and real-time control, will not send monitor information to the monitor center again and only send message when telemeter station is unable to finish the task. Thus, GSM network only transmits the task message that telemeter Agent union unable to finish and need monitor center to finish, thereby reduce the communication amount of GSM network greatly, improve communication efficiency; At the same time the telemeter station will not wait the control information of monitor center again, improve the real-time character of the long-range monitoring system of irrigated area greatly. 


\section{AGENT AND MULTI-AGENT}

Generally speaking, Agent is one kind independent calculation entity or procedure that can perceive the environment under specific social environment, and can realize a series of design objects by flexible and independent operate. As independent individual, under urged by certain goal, Agent has certain self- control ability of it's own behavior and inside state, and understand users' true intention as accurate as possible, adopt the positive behavior, effectively utilize various kinds of data, knowledge, information and calculation resource that can utilize in the environment, offer fast, accurate and satisfied services to user. (Zhang, 2004)

Multi-Agent systems is one computing system that one group Agent finishes some tasks or achieve some goals through cooperating, these Agents should cooperate and solve the problem that exceed each individual ability, they independent and distributed to run, and coordination and service each other, the goal and behavior between Agents contradiction and conflict, which is coordinated and solved through the means such as competition or consulting, to finish a task together. The important of Multi-Agent systems is the coordination of intelligent behavior between independent Agent, coordinate one's own knowledge, goal, skill, and scheme between them to produce the corresponding behavior or solve the problem. While the question is solved, for a common goal, these Agents share relevant knowledge of questions and solving method. Multi-Agent system requires system the exchange of every Agent has intelligence or self-organize ability (For instance reasoning, planning, studying, etc.) (Herry, 2006; Mohamed, 2007; Thibaud, 2007)

\section{LONG-RANGE MONITORING SYSTEM OF IRRIGATED AREA BASED ON MULTI-AGENT AND GSM}

\subsection{Systematic structure}

The long-range monitoring system of irrigated area is generally make up of the monitor and manage center, telemeter station and communication network between them. The monitor and manage center is a key part of monitoring system, its task can divide into many son tasks, so in the longrange monitoring system of irrigated area based on Multi-Agent and GSM, structured a monitor Agent union formed by a lot of Agent; In same way, the 
task of telemeter station also can divide into many son tasks, so structured a telemeter station Agent union; To counter the dispersiveness that telemeter station distribute, this system adopt GSM wireless network to realize the communication between monitor center and telemeter station, the concrete system structure is as Fig. 1 shows (Zhao, 2007).

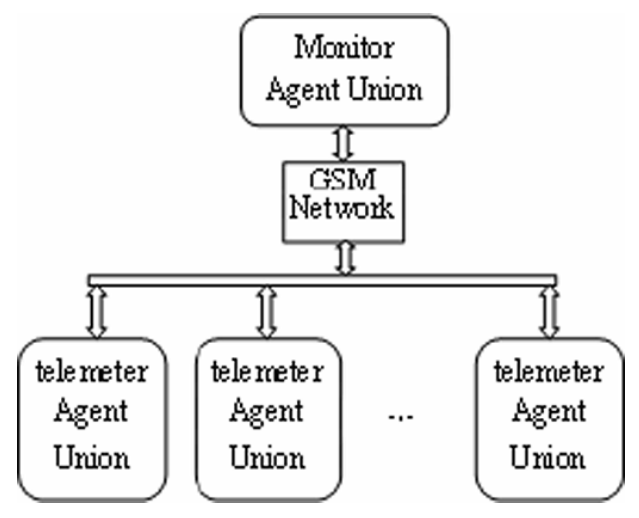

Fig. 1. Monitor system structure chart

\subsection{Classification and function of Agent}

Can be found from the systematic structure chart, the long-range monitoring system of irrigated area based on Multi-Agent and GSM make up of monitor Agent union and telemeter Agent union these two kinds of Agent unions and GSM communication networks, the important part used to finishing system task is two kinds of Agent unions. Each of These two kinds of Agent unions is composed of a lot of Agents, each one Agent has one's own tasks, and the completion of their task is serves for the completion of the overall task of the union.

\subsubsection{Subdivision of the indicators}

The monitor Agent union is a monitor center of the whole system, it is make up of one manage Agent and a lot of function Agents, the concrete structure is as Fig. 2 shows. Manage Agent1 (M-Agent1) manage the distribution of monitor center inner task and carries on communication with the external world; Information storage Agent1 (IS-Agent1) is used for storing the concretely information of water proportion of all telemeter station; Calculate Agent1 (C-Agent1) is used to calculate the water quantity distribution among a lot of telemeter stations; Data store Agent1 (DSAgent1) is used to store the result that C-Agent1 calculated for after using. If there are data in the monitor center need to output (type), it is may add another data output Agent (DO-Agent). 


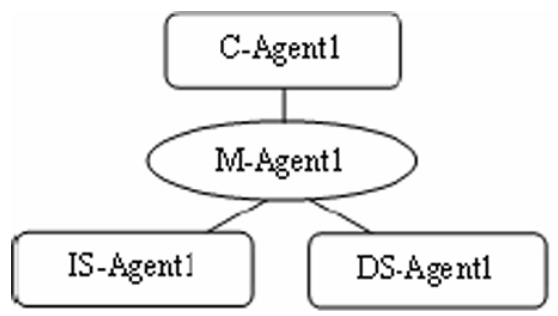

Fig. 2. Monitor agent union structure chart

\subsubsection{Telemeter station Agent union}

The telemeter station Agent union is make up of one manage Agent and a lot of function Agents too. Manage Agent2 (M-Agent2) responsible to distribute and resolve the task inside telemeter station and carry on communication with the external world. Information store Agent2 (ISAgent2) is used for storing the relationship curve information of the water level with the gate level; The data store Agent2 (DS-Agent2) is used for storing the concretely relationship data of the water level with the gate level; Calculate Agent2 (C-Agent2) is used for calculating the relation of the water level and the gate level; The water level monitor Agent1 (WLMAgent1) is used for monitoring the water level and obtaining water level information; Gate monitor Agent1 (BLM-Agent1) is used for monitoring the gate level and obtaining the gate level information; Operate control Agent1 (OC-Agent1) is used for controlling the opening and close of the gate. For the validity of communication, the communication inside the telemeter station Agent union is all finished through M-Agent2. The concrete structure is as Fig. 3 shows

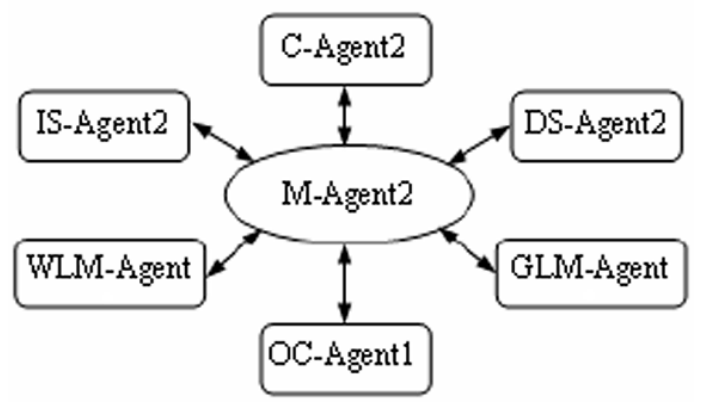

Fig. 3. Telemeter agent union structure chart 


\subsection{Air quality assessment based on extension of matter-element model}

The key of the communication coordination within the long-range monitoring system of irrigated area based on Multi-Agent and GSM is the coordination communication between monitor Agent union and telemeter station Agent union.

Because the telemeter station of irrigated area is distributed more scattered, use the wired network though communication is high efficiency, but it is very difficult to concretely implement, so adopt GSM wireless network to carry on communication. Monitor Agent union and telemeter Agent union all with one manage Agent, each manage Agent is a administrator inside one's own union, responsible for coordinating communication with external besides responsible for distributing the union inside task and communication coordination among Agent, so the communication coordination of the whole big system is the communication coordination between manage Agent1 in monitor Agent union and manage Agent2 in telemeter Agent union. The communication of the wireless network is different from wired network, in order to realize communication, need to set up a communication and control module within each two kinds of management Agent, as the data communication interface of GSM network.

At present, communication agreement interface adopted by mobile communication of GSM is the AT order collection, which norm is had described in detail by GSM07.05 standard and GSM07.07 standard of ETSI standard, in the world all mobile communication equipment terminal such as short news terminal module, GSM cell-phone support above standards at present. Sending and receiving SMS message has two ways: Text Mode (the mode of text) based on AT order collection and PDU (protocol description unit) mode based on AT order collection. Because the mode of the text is simple and easy to realize, and does not need to encode and decoding, so the communication between two kinds of manage Agent adopts the mode of the text to carry on the communication.

\subsection{Air quality assessment based on extension of matter-element model}

As to this system, the total task is to distribute water quantity according to the demand of water quantity of each telemeter station, and carry on the result of this distribution. It is can be find out from the above discussion, every function Agent finishes the task of subsystem under coordination of 
manage Agent, and the subsystem and subsystem finish the transmission of the task information between them through the communication of GSM network, the concrete realization step is as follows:

Step1: M-Agent1 receives this task of water amount allotment from the network of rivers (or the message of water amount allotment imported by administrative staff), and send water amount allotment information to DSAgent1;

Step2: DS-Agent1 inquires in its database according to the information of water amount allotment, and sends the result inquired to M-Agent1;

Step3: M-Agent1 receives the information, and analysis, if there is corresponding information, send information to M-Agent2, carry out Step9; Without, send a request of accessing information to IS-Agent1;

Step4: After received the request, IS-Agent1 accesses the proportion information of the water amount allotment of all gates and send to MAgent1;

Step5: M-Agent1 send the information of water amount allotment of all gates and the information of overall water amount allotment to C-Agent1;

Step6: C-Agent1 calculates the allocation program and sends to $\mathrm{M}$ Agent1;

Step7: M-Agent1 sends the information of new allocation program of water amount to M-Agent2, carries out Step9; Send this information to DSAgent 1 at the same time;

Step8: DS-Agent1 stores the new allocation program of water amount in its database for accessing next time;

Step9: M-Agent2 send the information of water amount to DS-Agent2;

Step10: DS-Agent2 inquires in its database according to the message received, sees whether there is a corresponding water level - gate level relation data, if it is exist, access this information and send to M-Agent2; If it is not exist, send the message of "without" to M-Agent2;

Step11: M-Agent2 receives the reply message of DS-Agent2, and analysis, if there is corresponding information, send the corresponding relation data of water level - gate level to OC-Agent1, namely carry out Step15; Without, send water amount information to C-Agent1, namely carry out Step12;

Step12: C-Agent2 receives the water amount information, and calculates the relation of water level - gate level, then send the result of calculation to M-Agent2;

Step13: M-Agent2 receives the result of calculation by C-Agent2 and sends it to OC-Agent1 carries out Step15; and send the result of calculation to DC-Agent2, carry out Step14; 
Step14: DS-Agent2 stores the result of calculation received in its database for accessing next time;

Step15: OC-Agent1 controls the opening and close of the gate according to the received relation data of water level - gate level;

Step16: After the gate is opened, WLM-Agent1 and GLM-Agent1 begin to run, and the data monitored are sent to M-Agent2;

Step17: M-Agent2 sends the data to C-Agent2;

Step18: C-Agent2 calculates out water level - gate level relation curve and relation data, and returns the result to M-Agent2;

Step19: M-Agent2 sends the relation curve information to IS-Agent2;

Step20: IS-Agent2 compares the new relation curve with already existing, if the result is same, do not send reply information, if does not agree, store new relation curve information in its database, and send the result to $\mathrm{M}$ Agent2;

Step21: After M-Agent2 received the reply of IS-Agent2, send the new relation data of water level-gate level to OC-Agent1, carry out Step15; Send to DS-Agent too at the same time, carry out Step22;

Step22: DS-Agent stores the new relation data of water level - gate level in its databases.

\section{CONCLUSIONS}

Multi-Agent system is composed of a lot of Agents, finishes the systematic task common and intelligence through the communication coordinating among them; GSM network is the wireless communication networks that is modern generally adopted. The long-range monitor system of irrigate area built by combine Multi-Agent system theory and GSM network together has a lot of advantage: at first, the monitor center forms a Agent union, finish its inside task by a lot of Agent through negotiation, instead of be finished by staff members through coordinated; Secondly, telemeter station (spot) construct a Agent union too, has more functions use it to replace the simple monitor equipment, some problems which can be solved inside the telemeter station no longer send information to the monitor center, thus reduce the communication to bear; Finally, the ones that communicate are not again the monitor center and telemeter station (spot) through GSM, but only manage Agent within them, communication amount reduces greatly, communication is more effective and swift.

It can find out through the test data, the application of the long-range monitoring system of irrigated area based on Multi-Agent and GSM can greatly reduce losses of water quantity, thus can greatly reduce peasants' water expenses burden. This system is low costs, convenient to safeguard, with complete function, and has the functions such as real-time control gate 
and data gathering, storing, dealing with and automatic yielding water quantity, etc. in real time, overcome the phenomenon such as difficult to macroscopic distributing water quantity, bad to regulating water quantity in real time, reduce work intensity, economize the valuable water resource.

\section{ACKNOWLEDGEMENTS}

In the course of research of this system, I have been supported vigorously by the fluid institue of lanzhou University of technology, especially got the help of the teachers of the engineering department of water conservancy and power, such as Xu cungdong, Wangzhijun and Biguiquan etc. In the stage of test and verify, I have got support and help of all staffs of one management office of one administration bureau, express seriously thank to their support and help in here!

\section{REFERENCES}

Herry Purnomo, Philippe Guizol, Simulating forest plantation co-management with a multiagent system, Mathematical and Computer Modelling, Vol. 44. No. (5-6). 2006. pp. 535-552

Mohamed Salah Hamdi, A multi-agent approach to information customization for the purpose of academic advising of students. Applied Soft Computing, Vol. 7. No. 3. 2007. pp. 746-771

Thibaud Monteiro, Daniel Roy, Didier Anciaux, Multi-site coordination using a multi-agent system, Computers in Industry, Vol. 58. No. 4. 2007. pp. 367-377

Zhang jie, Gao liang, Li pei-gen, The application of Multi-Agent technology in the advanced manufacture, Science Press of China, 2004.

Zhang Kun-ao, Application of wireless local area network in interconnection of the monitoring system for large irrigating area, Journal of Xi'an University of Science \& Technology, Vol. 25. No. (4). 2005. pp. 86-87

Zhao ting-hong, Qi xue-yi, Control System of Hydropower Economic Running Based on Multi-Agent Theory, Journal of Micro-computer information, Vol. 23. No. (4-3). 2007. pp. 54-56 